Vol. 62 (2000) [347-349]

\title{
Infra-solvmanifolds of dimension four
}

\author{
Robin J. COBB
}

In dimension four it has been shown that the task of classifying compact 4-manifolds up to diffeomorphism is not recursive [2]. Nevertheless much effort has been expended into the classification of certain classes of compact 4-manifolds yielding good insight into their nature. In particular, in light of the fact that geometric structures in the sense of Thurston (see $[7,6]$ ) have led to a classification of manifolds in dimensions 1 and 2 , and possibly 3 , there has been much interest in the geometric manifolds of dimension four.

There are nineteen different geometries of dimension four, one of which is an infinite family. Our interest is with the six infra-solvmanifold geometries, which includes the infinite family. Loosely speaking our concern is with those compact 4-manifolds that are the quotient of some simply connected solvable Lie group $S$ by an isometric action of a group $\Gamma$ (see $[1,5]$ ). When $S$ is Abelian and $\Gamma$ is discrete the resulting manifolds are flat, and there are only finitely many diffeomorphism types in each dimension. Moreover the diffeomorphism class of a flat manifold is completely determined by the isomorphism class of its fundamental group (Bieberbach Groups). When the Lie group $S$ is nilpotent and $\Gamma$ is discrete, the associated manifolds are referred to as infra-nilmanifolds. Although this class of manifolds is much larger than that of the flat manifolds (in the sense that there are infinitely many infra-nilmanifolds in each dimension) they are similarly classified up to diffeomorphism by the isomorphism class of their fundamental groups. Moreover these groups (Almost-Bieberbach Groups, see [4]) are algebraically determined by analogous criteria to those for flat manifold groups.

When $S$ is solvable it has been shown that the isomorphism class of the fundamental group of such a manifold determines the diffeomorphism class in all dimensions with the possible exception of dimension four [5]. In Chapter 2 of the thesis this result is extended to include all 4-dimensional compact infra-solvmanifolds with the possible exception of one anomalous class. This (possibly empty) exceptional class of compact infra-solvmanifolds consists of those 4-manifolds with virtually nilpotent fundamental groups that do not admit any discrete presentations.

Received 13th April, 2000

Thesis submitted to The University of Sydney, June 1999. Degree approved, November, 1999. Supervisor: Dr. Jonathan Hillman.

Copyright Clearance Centre, Inc. Serial-fee code: 0004-9727/00 \$A2.00+0.00. 
The fundamental groups of those compact infra-solvmanifolds with a 4-dimensional model have been essentially classified with the exception of two models (one of which belongs to the infinite family). In Chapter 3 of the thesis the first effort towards the classification of the fundamental groups of infra-solvmanifolds admitting one of the remaining two models is made.

Central to the theory and methods presented here are various bundle-structures and foliations (Seifert Fibrations) associatd with the manifolds $M$ in question. These structures permit us to algebraically construct $\Pi_{1}(M)$ as group extensions of geometric manifold-groups of lower dimensions. Moreover a bundle-structure on a manifold with geometric base and fibre can endow the total space with a geometric structure of its own. Chapter 4 of the thesis completes the work initiated by Ue [8]: in classifying those total spaces of surface-bundles over surfaces with flat base and fibre (up to bundle-diffeomorphism) and thence showing that each such manifold admits an infrasolvmanifold geometry.

In addition, during his Ph.D. candidature the author in collaboration with his Ph.D. supervisor has produced the publication [3]. Here it is shown that those 4-manifolds that arise as total spaces of $S^{2}$-bundles over closed, connected aspherical surfaces are geometric of type $S^{2} \times E^{2}$ or $S^{2} \times \mathbb{H}^{2}$. Conversely, it is shown that any 4-manifold $M$ admitting one of the above two geometries is the total space of such a sphere-bundle precisely when its fundamental group $\Pi_{1}(M)$ is torsion free. Analogous results are also obtained for total spaces of $\mathbb{R}^{2}$-bundles over aspherical surfaces.

\section{REFERENCES}

[1] L. Auslander, 'An exposition of the structure of solvmanifolds', Bull. Amer. Math. Soc. 79 (1973), 227-285.

[2] W.W. Boone, W. Haken, V. Poénaru, 'On recursively unsolvable problems in topology and their classification', in Contributions to Mathematical Logic, (H. Arnold Schmidt, K. Schütte and H.J. Thiele, Editors), Proceedings of the Logic Colloquium, Hannover 1966 (North-Holland, Amsterdam, 1968), pp. 37-74.

[3] R.J. Cobb and J.A. Hillman, ' $S^{2}$-Bundles over aspherical surfaces and 4-dimensional geometries', Proc. Amer. Math. Soc. 125 (1997), 3415-3422.

[4] K. Dekimpe, Almost-Bieberbach groups: Affine and polynomial structures, Lecture Notes in Mathematics 1639 (Springer-Verlag, Berlin, Heidelberg, New York, 1996).

[5] F.T. Farrell and L.E. Jones, 'Compact infrasolvmanifolds are smoothly rigid', in Geometry from the Pacific Rim (Walter de Gruyter, Berlin, 1997), pp. 85-97.

[6] G.P. Scott, 'The geometries of 3-manifolds', Bull. London Math. Soc. 15 (1983), 401-487.

[7] W.P. Thurston, 'Three dimensional manifolds, Kleinian groups and hyperbolic geometry', Bull. Amer. Math. Soc 6 (1982), 357-381.

[8] M. Ue, 'Geometric 4-manifolds in the sense of Thurston and Seifert 4-manifolds, I', J. 
[3]

Math Soc. Japan 42 (1990), 511-540.

School of Mathematics and Statistics The University of Sydney

Sydney NSW 2006

Australia

e-mail: robinc@maths.usyd.edu.au 